\title{
Aerodynamic Performance of Natural Laminar Flow Aerofoils Applied to Low- and High-Speed Wings
}

\author{
Ramón López Pereira*,2, José Omar Martínez Lucci², and Fermín Navarro Medina ${ }^{3}$ \\ * SAFRAN Engineering Services GmbH, Donauwörth, Germany \\ 2 Department of Mechanical and Aerospace Engineering, Universidad Europea de Madrid, Villaviciosa de Odón, Spain \\ ${ }^{3}$ Department of Mechanical Engineering, Thermal Machines and Engines and Fluids, Universidad de Vigo, Vigo, Spain \\ E-mail: ramon.lopez-pereira@safrangroup.com (ramlopper@gmail.com), \\ joseomar.martinez@universidadeuropea.es, fermin.navarro.medina@uvigo.es
}

\begin{abstract}
The aim of this investigation is to assess if the use of Natural Laminar Flow (NLF) aerofoils alone improves the efficiency of a wing in comparison to their NACA equivalents.

To compare performance, the lift-to-drag ratio (Efficiency) of the wings has been considered. A cruise speed range from M0.1 to M0.3 was analysed for the low-speed wing, while a Mach sweep between M0.3 and M0.85 was assessed for the high-speed wing. A final analysis was performed to evaluate the effect of the presence of a sweepback angle in the wing, from straight leading edge to a 10-degree-sweepback wing.

For the low-speed wing, it was observed that the efficiency of the laminar wing is slightly decreasing with speed (up to $17 \%$ between M0.1 and M0.3) but is increasingly higher than in the NACA wing (from $5 \%$ at M0.1 to $16 \%$ at M0.3). This means that the aim of the laminar aerofoil is met, so that for a cruise speed between 100 and $300 \mathrm{~km} / \mathrm{h}$ lower drag is produced and therefore lower thrust (and fuel consumption) is required.

In the case of the high-speed application, it was found that the laminar flow wing had lower efficiencies when compared to its NACA equivalent. Analysing the results, it was noted that the ratio of the lateral forces to lift had a direct relation to the efficiency: when this ratio was increased, the efficiency was decreased, and vice-versa. It was observed that, in the case of laminar wings, not applying a sweepback to the leading edge (LE) is optimal and duplicates the efficiency with respect to adding any angle. Moreover, this is the only case observed where the efficiency of the laminar wing is higher than its NACA equivalent.
\end{abstract}

Keywords: Environmentally friendly technology; laminar flow; low-speed; high-speed; aerodynamic efficiency; performance

\section{Introduction}

Aircraft design has always tried to put the airlines at focus. This means that the wishes of the airlines need to be considered. Previously, this meant bringing more passengers further and faster, but the current path is to have greener aircraft, that is, the emissions of carbon and nitrogen oxides should be reduced as well as the noise made by the aircraft. This wish is not only driven by the airlines' corporate social responsibility guidelines, which is also the case, but predominantly due to more restrictions in terms of regulation, mainly in Europe, aiming for a cleaner sky [1].

Even though the main focus in this cleaner sky approach has been set for engine manufacturers (either directly by new regulations or by the aircraft builders), the main competitors in the aircraft-manufacturing field have been receiving pressure from the market to release improvements towards the clean sky objective.
This investigation tries to assess if the use of NLF aerofoils provides real benefit in terms of drag reduction or if it is still non-reliable for this use. A new design of high-speed NLF aerofoil is included to try to optimize its performance. Even though the usual wing design will include several aerofoils inside it, for the purpose of this research it was chosen to use a single aerofoil spanwise.

As a first approach, standard wing parameters have been used on both aerofoil types, and then a wing sweepback angle parametric analysis have been performed in order to assess its effect on high-speed wings. In the case of low-speed wings, the selected aerofoils are a NASA-designed NLF(1)-1015 aerofoil [2] and its 4-digit NACA equivalent (NACA4615), while for the high-speed case a new aerofoil has been designed (named Raw Laminar Profile for High Speed Flow, RLPHSF) and its NACA equivalent (NACA1709) has been compared against it. 
To compare the performance of the wings, the efficiency has been obtained at different cruise conditions for each case. In the low-speed designs, this has been selected as $4900 \mathrm{~m}$ (FL160) International Standard Atmosphere (ISA) and a speed range between M0.1 (30m/s, 60KTAS) to M0.3 $(95 \mathrm{~m} / \mathrm{s}, 185 \mathrm{KTAS})$. For the high-speed wings, two sweeps are performed as indicated before, though both at $11000 \mathrm{~m}$ ISA (FL360). A speed variation between M0.3 $(90 \mathrm{~m} / \mathrm{s}$, 175KTAS) and M0.85 (250m/s, 485KTAS) was considered for the standard design, while a fixed M0.77 $(230 \mathrm{~m} / \mathrm{s}$, 445KTAS) condition with LE-sweepback angles ranging from zero to 10 degrees were also analysed.

\section{Methodology}

All cases have been modelled using the Lattice Boltzmann software XFlow. Dassault Systèmes XFlow [3] is a software package of new generation CFD, differing from classical CFD (e.g. ANSYS CFX) in that it uses the Lattice Boltzmann approach (transient and particle-based), instead of a Reynolds-Averaged Navier-Stokes (RANS) modelling. This software has been validated for benchmark problems in different industries, including the aeronautical field [4]. It can solve two- and three-dimensional problems indistinctively.

The time consumption in a full simulation (from setup to postprocessing) is greatly reduced with the use of XFlow, as the pre-processing (including meshing) is much simpler. If wake effects want to be studied in detail, XFlow also introduces a dynamic meshing, which will follow the wake for further time steps. The last and main reason why this software has been used for this investigation is that turbulence need not be modelled, but solved, without a major increase in solver time. This eliminates the error-inducing situation of having to choose the appropriate turbulence model, which is of capital importance when considering the use of NLF aerofoils.

Several setup parameters can be considered when preparing the simulations in XFlow and will be discussed hereafter. These can be classified in solver and boundary conditions parameters.

\subsection{Solver parameters}

Among the several parameters that can be considered in terms of solver in XFlow, there is the main reason for selecting this software: turbulence modelling. Although turbulence can be solved as a Direct Numerical Simulation (DNS), for typical examples where other turbulence models have been validated, XFlow offers the possibility of using the following:

- Spalart-Allmaras: a one-equation model used for low-Reynolds and that is implemented in most CFD tools

- Smagorinsky / Dynamic Smagorinsky: these methods model the kinematic viscosity using a filter scale and the Smagorinsky constant. While the regular Smagorinsky method keeps the constant invariable throughout the simulation, the dynamic version adapts the values depending on the solutions that it obtains in the different steps.
- Wall-Adapting Local-Eddy (WALE): this is the standard setup option in XFlow, as it has been proven to be accurate both near and far from walls to simulate boundary layers.

Another setup condition for the solver is the definition of the fluid state equations, where the user should decide between:

- Incompressible: the option to be chosen for low speeds and fluids that are considered constant in density. This option was used for the low-speed wing models, as for up to M0.3 incompressibility can be considered to be acceptably accurate.

- Ideal gas: modelling the density using the ideal gas equation. This method was used in the case of the high-speed wings, as the analyses are mainly aimed at inlet velocities above M0.5.

- Boussinesq model: using the Boussinesq approximation for density, which requires the thermal expansion coefficient to be defined and should only be applicable in combination with a thermal model, as otherwise the results would be the same as using the incompressible approach.

Finally, the thermal modelling (that is, if the energy equation is used or not) needs to be defined. Out of the different options offered by XFlow, the isothermal approach was used for the low-speed wings, where the energy equation is not resolved. In the case of the high-speed wings, the coupled energy method was considered, where compressibility is considered for the energy equation. Two additional options are available, the segregated energy (similar to the coupled energy but not considering compressibility effects) and a supersonic model, which is still under validation and therefore was not considered although it would be the most accurate for transonic flight conditions.

Additionally, several viscosity models can be considered. In the case of the low-speed wing, a regular Newtonian viscosity model (temperature independent) has been considered as the energy equation was not used, while for the high-speed wings the Sutherland model using standard values for the constants required for modelling of standard air $(C=120 \mathrm{~K}$; $\mathrm{T}=291.15 \mathrm{~K}$ and $\mu=18.27 \cdot 10^{-6} \mathrm{~Pa} \cdot \mathrm{s}$ ) was taken.

\subsection{Boundary conditions parameters}

XFlow uses a "Non-equilibrium wall function" to model the boundary layer on the analysed surfaces. This means that the adverse pressure gradients are taken into consideration and the transitional effects (laminar to turbulent, detachment and reattachment) can be observed in the simulation, and as previously indicated turbulence effects are solved. The modelling options available allow a near-wall refinement and a wake-following refinement, in which the calculation points (the equivalent to the mesh in a RANS CFD) will be modified at each step depending on the results of the previous simulation step results. Moreover, a general resolved scale is defined which indicates the distance between calculation points in the free stream conditions. 
The walls need thermal exchange boundary conditions if the energy equation is considered. The options available for XFlow include adiabatic, temperature law, heat flux, free convection and volume heat source. As in both wings no thermal exchange was taken into account, the adiabatic model (no heat flux through the solids) was used.

\section{Low-speed model definition}

Different aerofoils have been considered for this design. It was found that a moderately higher curvatures combined with thicknesses of over $10 \%$ were drawing the best efficiencies at levelled flight. This also produces setbacks in terms of range of pitch and critical Mach [5], but the analysis conditions for the performance comparison are levelled flight (no pitch) and low speed (thus far from the critical conditions). The selected aerofoil is the NASA-developed NLF(1)-1015, which has a maximum thickness of $15 \%$ at $40 \%$ chord and a maximum camber of $4 \%$ at $63 \%$ chord, distributed as shown in fig. 1. This means that the 4-digit NACA equivalent of this aerofoil would be the NACA4615, which is shown in direct comparison with the NLF(1)-1015 in fig. 2.

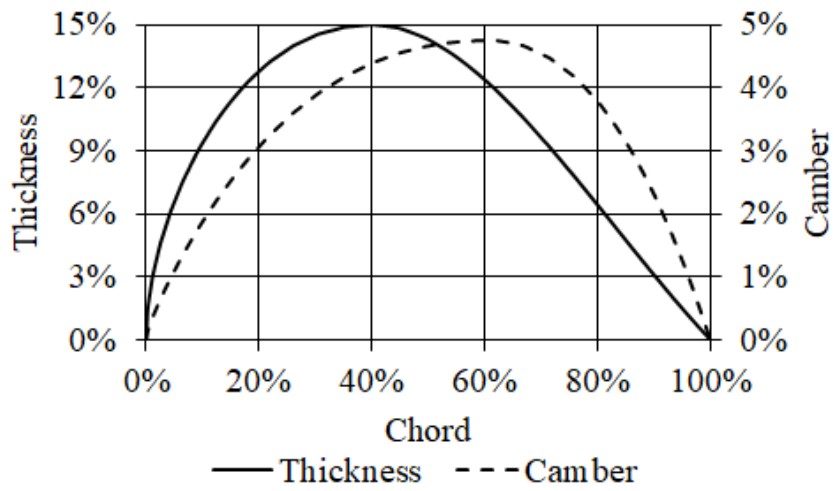

Figure 1: Thickness/Camber distribution for NLF(1)-1015

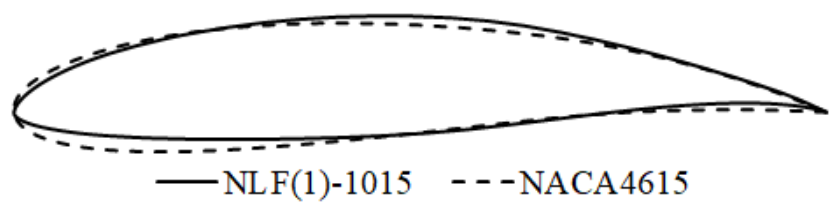

Figure 2: Aerofoil Comparison: NLF(1)-1015 vs NACA4615

The wingspan has been defined as $13.7 \mathrm{~m}$, with a taper of 0.5 and a surface of $19 \mathrm{~m}^{2}$, which is representative of commuter aircraft (Part 23 certification). As usual in this type of aircraft, no sweepback angle nor twist or dihedral are introduced in the wing. Finally, no winglet has been considered. The final design of the wing can be seen in fig. 3 .

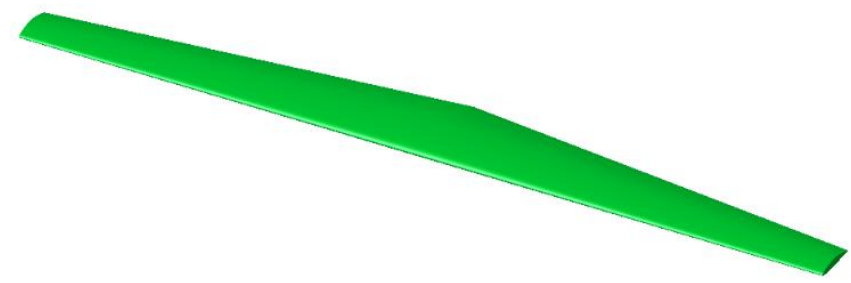

Figure 3: Low-Speed Wing
For these analyses, the Isothermal (non-compressible flow) model has been used, with the air conditions set at ISA, $4900 \mathrm{~m}$ above sea level. Turbulence has been resolved and not modelled, and a scale (grid size) of $20 \mathrm{~cm}$ was used overall except near the wing, where it was refined to $10 \mathrm{~cm}$. The simulation time was set to $2 \mathrm{~s}$, with a time step of $0.02 \mathrm{~s}$. The flight speeds have been selected ranging between M0.1 $(30 \mathrm{~m} / \mathrm{s}, 60 \mathrm{KTAS})$ to M0.3 $(95 \mathrm{~m} / \mathrm{s}, 185 \mathrm{KTAS})$, in M0.05 steps.

\section{High-speed model definition}

In the case of the high-speed NLF aerofoils, not many have been designed yet and the geometrical details would probably not be undisclosed. Following the regular considerations for high-speed aerofoil design, though, it can be agreed that lower curvatures and thicknesses are the most efficient, and therefore the most used [6]. The main concerns are that at low speeds (e.g. take-off and landing) the use of non-laminar high-lift devices like flaps and/or slats is required, but for the analysed levelled, cruise conditions this is negligible. A new design has been developed considering the previous conditions, and was named RLPHSF. The maximum thickness used is $9 \%$ at $30 \%$ of the chord, with a maximum camber of $1.5 \%$ at $72 \%$ of the chord; the distribution of both parameters along the chord can be seen in fig. 4 . Hence, the 4-digit NACA equivalent of this aerofoil is the NACA1709, and a comparison between both aerofoils is shown at fig. 5 below.

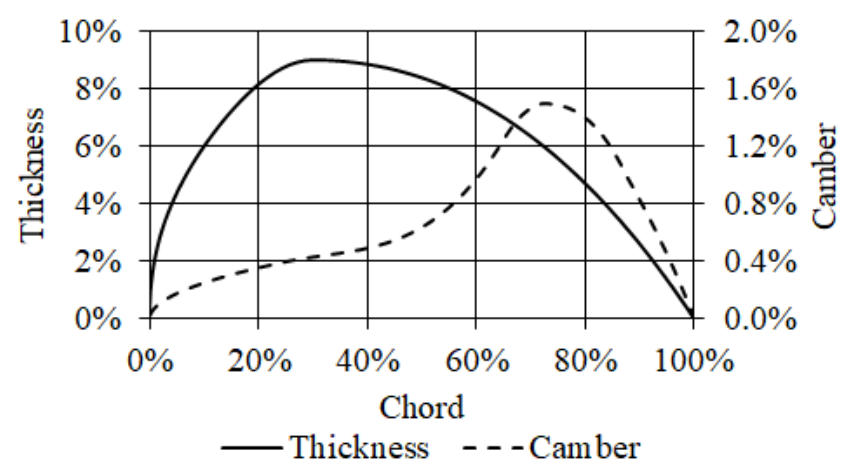

Figure 4: Thickness/Camber distribution for RLPHSF

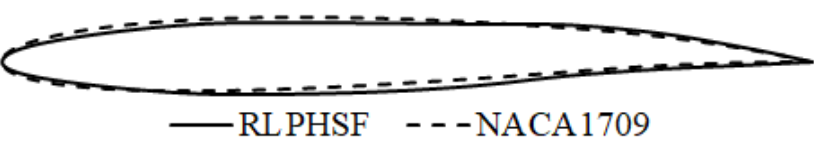

Figure 5: Aerofoil Comparison: RLPHSF vs. NACA1709

There are two analysis sweeps performed for this wing, a fixed-design flight-condition sweep and a fixed-flightcondition sweepback-angle modification keeping all other geometrical parameters fixed. For all cases, the wingspan has been defined as $26.3 \mathrm{~m}$, with a taper ratio of 0.6 and a wing surface of $69 \mathrm{~m}^{2}$, which is representative for business jets (Part 25 certification). 
In the fixed-design case, a sweepback angle of 25 degrees without twist or dihedral has been introduced. The winglet was defined as $10 \%$ of the wingspan, with a dihedral of 10 degrees and an internal taper of 0.5 . The final design of the wing can be seen in fig. 6 .

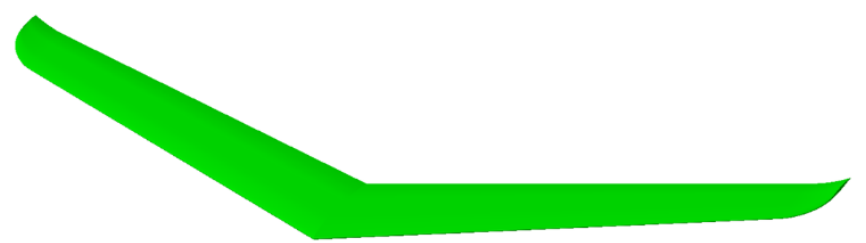

Figure 6: High-Speed Wing

Finally, using the same basic geometrical parameters but removing the winglet, analyses with sweepback angles between zero and 10 degrees in 1.25 degrees steps have been considered.

For these analyses, the coupled energy (isentropic) model has been considered, with the air conditions set at ISA, 11000m above sea level. Turbulence has been resolved, and a scale (grid size) of $20 \mathrm{~cm}$ was used overall except near the wing, where it was refined to $10 \mathrm{~cm}$, as it was in the previous cases. The simulation time was set to $2 \mathrm{~s}$, with a time step variating depending on the analysis Mach. This is changed due to the requirement for the time step to be able to accommodate the movement of the air between two analysis points inside it. This means that, if the time step is too high, a tracked particle would have gone through more than a single analysis point and therefore the results would not be accurate. A speed variation between M0.3 (90m/s, 175KTAS) and M0.85 $(250 \mathrm{~m} / \mathrm{s}, 485 \mathrm{KTAS})$ was considered for the fixed-geometry wing, while M0.77 $(230 \mathrm{~m} / \mathrm{s}, 445 \mathrm{KTAS})$ was taken for all sweepback variation analyses.

\section{Low-speed results}

The results for the low-speed wings show that, for any of the analysed Mach conditions, the NLF wing shows improved efficiency. Even though its value drops for the NLF wing as the speed is increased (as reflected in fig. 7), it can also be observed that the difference in efficiency between using the NACA and the NLF aerofoil is actually increased from roughly $5 \%$ at M0.1 to over $15 \%$ at M0.25 as shown in fig. 8 . Moreover, it can be concluded that, on traditional designs of commuter aircraft, the use of NLF aerofoils can reduce the drag produced by the wing, and therefore the required thrust.

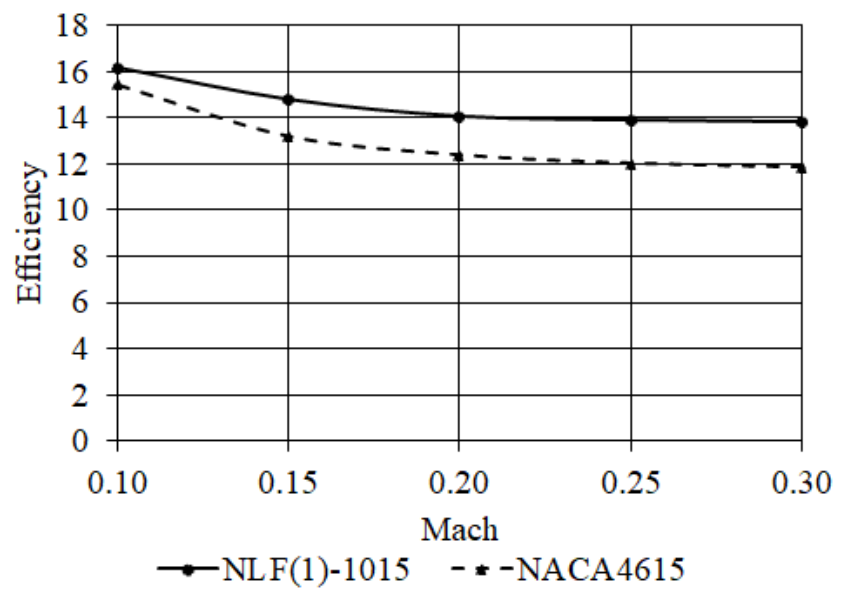

Figure 7: Efficiency for the low-speed wings

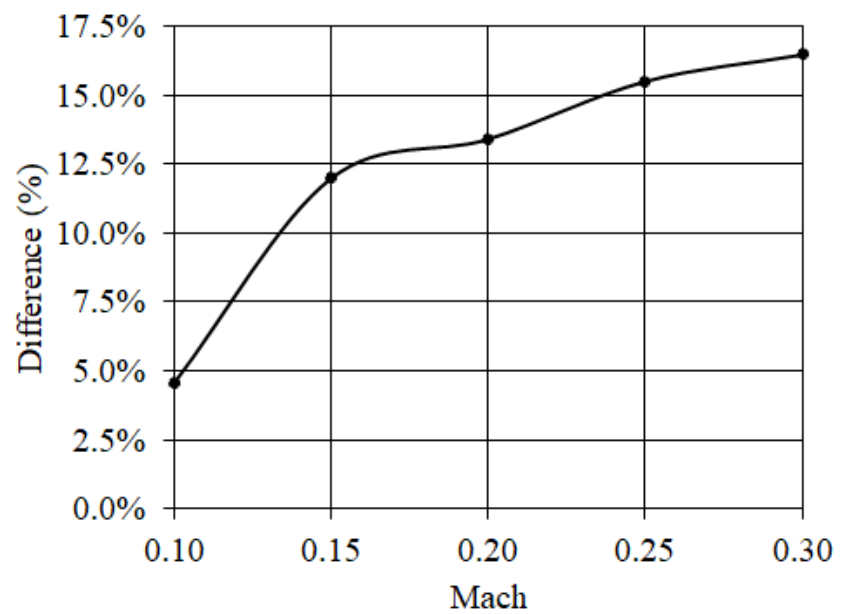

Figure 8: Difference in efficiency between the low-speed wings

\section{High-speed results - fixed wing design}

In the case of the high-speed application, the NLF wing draws lower efficiencies when compared to its NACA equivalent. A deeper look into the results, not focusing only on lift and drag, shows that the ratio of the lateral forces to lift had a direct relation to the efficiency: when this ratio is increased, the efficiency is decreased, and vice-versa. This means that, in order to reach optimal efficiencies in high-speed wings, the lateral force needs to be restrained. Although this seems simple to comply with, the fact is that this lateral force is mainly produced by having a sweepback angle on the wing, which is usually required for flight at higher speeds [7]. It is noticeable and interesting to note that when the Mach increases from M0.6 onwards (up to near-transonic regions, as further analyses are not reliable) the efficiency is increased in the case of the NLF aerofoil. 


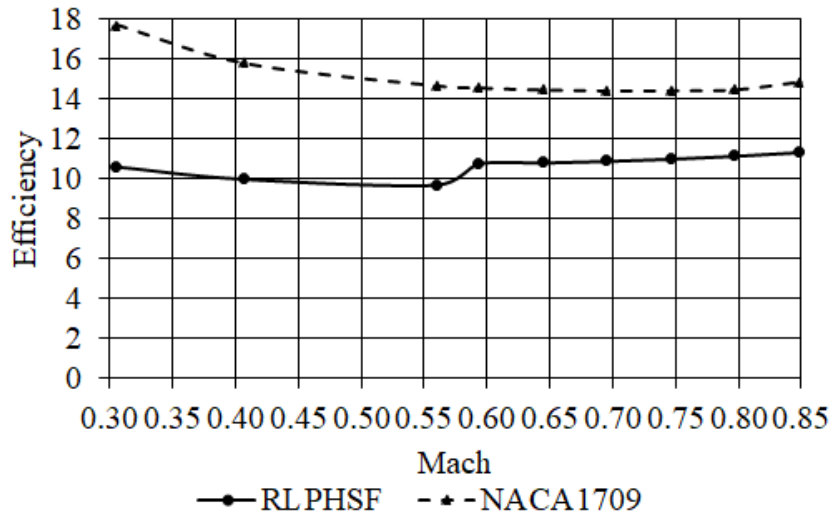

Figure 9: Efficiency for the high-speed wings, Mach sweep

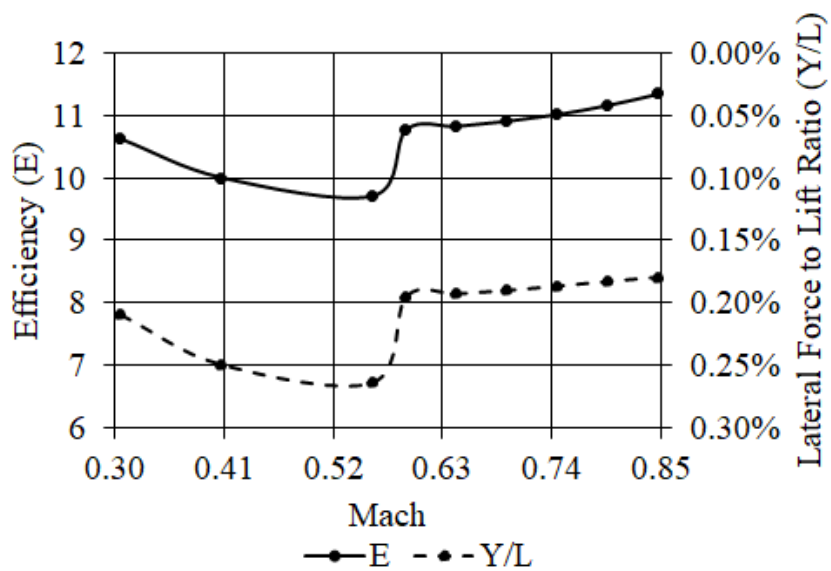

Figure 10: Relationship between lateral forces and efficiency for the high-speed NLF wing, Mach sweep

\section{High-speed results - Sweepback angle effect}

The final step on this investigation is to assess the effects of the sweepback on the efficiency of high-speed NLF wings. The results show that the efficiency at high speed is only higher for the NLF wing when no sweepback is present, dropping to less than half its initial value just by adding $1.25 \mathrm{deg}$ sweepback. As it can be assessed from fig. 11, there will be no significant differences between having low and high sweepback angles in terms of efficiency.

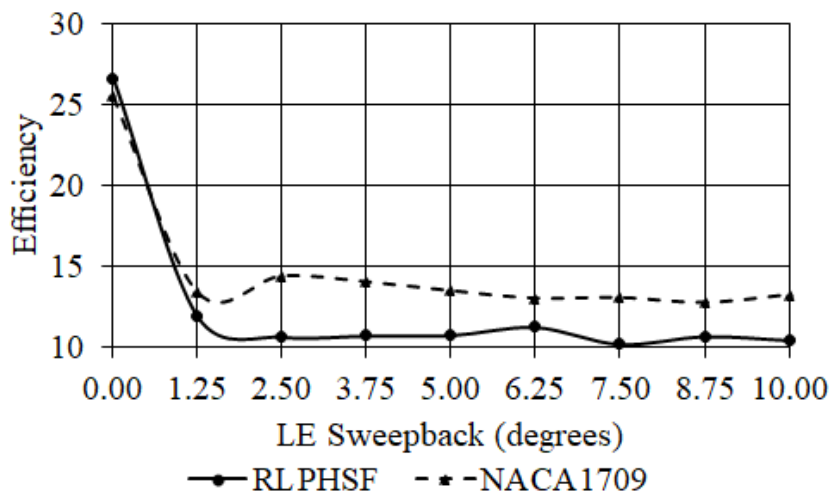

Figure 11: Efficiency for the high-speed wings, LE sweepback variation

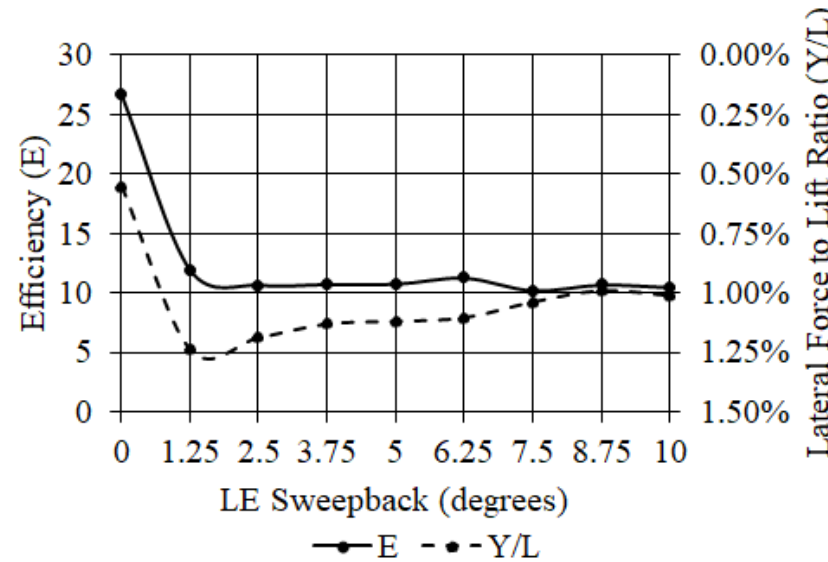

Figure 12: Relationship between lateral forces and efficiency for the high-speed NLF wing, LE sweepback variation

\section{Conclusions}

For Low-speed applications, it could be confirmed that the use of NLF aerofoils in a standard wing configuration is of advantage (up to $15 \%$ higher efficiency depending on the flight speed).

However, for a standard high-speed wing configuration (with sweepback), NLF aerofoils not only do not provide any improvement but show a $20 \%$ lower efficiency. This is seen to be due to the destabilizing effect of the lateral forces on the laminar boundary layer: for a concrete high-speed configuration, there is a direct relation between the lateral forces and the efficiency of the NLF wing when varying speed.

Finally, it was observed that, in the case of laminar wings, not applying a sweepback to the leading edge is optimal and duplicates the efficiency with respect to adding any angle at a high speed. Moreover, this is the only case observed where the efficiency of the laminar wing is higher than its NACA equivalent. 


\section{References}

[1] Horizon 2020 European Union Funding for Research \& Innovation, "Clean Sky," [Online]. Available: https://www.cleansky.eu/. [Accessed 0807 2019].

[2] M. D. Maughmer and D. M. Somers, "Design and Experimental Results for a High-Altitude, Longendurance Airfoil," Journal of Aircraft, vol. 26, no. 2, pp. 148-153, February 1989.

[3] Dassault Systèmes, "XFlow Brochure," 2019. [Online]. Available: https://www.3ds.com/. [Accessed 2606 2019].

[4] D. M. Holman, R. M. Brionnaud and Z. Abiza, "Solution to industry Benchmark Problems with the LatticeBoltzmann Code XFlow," in European Congress on Computational Methods in Applied Sciences and Engineering (ECCOMAS 2012), Vienna, 2012.

[5] J. D. Anderson, in Fundamentals of Aerodynamics, 5 ed., McGraw-Hill, 2011, pp. 728-737.

[6] C. D. Harris, "NASA supercritical airfoils: A matrix of family-related airfoils," NASA, 1990.

[7] A. Busemann, "Aerodynamischer Auftrieb bei Überschallgeschwindigkeit," in 5th Volta Conference, Rome, 1935. 